\title{
On Errors in Determinants
}

By I. M. H. Eitherington, Edinburgh University.

(Received 6th March, 1931. Received in Revised Form 6th March, 1932. Read 1st May, 1931.)

$\S 1$. Introduction. Care is needed in dealing with determinants whose elements are subject to experimental error, particularly when a determinant itself is small compared with its first minors. For, as these examples show, a relatively tiny error in one element may be responsible for a large error in the determinant :

$$
\left|\begin{array}{rrr}
-73 & 78 & 24 \\
92 & 66 & 25 \\
-80 & 37 & 10
\end{array}\right|=1 ; \quad\left|\begin{array}{rll}
-73 & 78 & 24 \\
92 & 66 & 25 \\
-80 & 37 & 10 \cdot 01
\end{array}\right|=-118 \cdot 94 .
$$

Consider a determinant $\Delta$, of order $n$, with elements $a_{i}$ $\left(i=1, \ldots, n^{2}\right)$. Let the actual error in each element $a_{i}$ be $e_{i}$, and let the resultant error in $\Delta$ be $E$. In $\$ \$ 2-4$, I shall find expressions for (i) $E$ in terms of $e_{i}$; (ii) the maximum range of $E$ in terms of the ranges of $e_{i}$; (iii) the probability distribution $P(E)$ of $E$ in terms of the probability distributions $p_{i}\left(e_{i}\right)$ of $e_{i}$, assumed independent and (for simplicity) symmetrical. In $\$ 5-7$, I shall investigate corresponding results for the quotient of two determinants which are identical except for one row or column. Such quotients are of importance in practical work, occurring in the solution of a set of simultaneous linear algebraic equations. In $\S 8$, the method is applied to an arbitrary function whose arguments are subject to error.

To apply the formulae to numerical determinants, it is necessary for first approximations to calculate the complete set of first minors; the second, third, etc., minors are required for closer approximations. The calculation of these minors is very laborious in the case of a determinant of large order. It may be pointed out, however, that all the first minors can be found in the course of evaluating a determinant and its adjugate by extant methods, for example that ${ }^{1}$ of T. Smith, Phil. Mag. (7), 3 (1927), 1007.

1 There is also a method suitable for use with a calculating machine, due to Dr Aitken, and as yet unpublished. 
§2. Actual error in a determinant. Let $A_{i}, A_{i,}, A_{i j k}, \ldots$ denote the first, second, third, .... minors of $\Delta$ corresponding to the elements indicated by the suffixes. An error $e_{i}$ in the term $a_{i}$ will cause an error $e_{i} A_{i}$ in $\Delta$; errors $e_{i}, e_{i}$ in the terms $a_{i}$, $a_{j}$, will together cause an error $\left(e_{i} A_{i}+e_{j} A_{j}+e_{i} e_{j} A_{i j}\right)$ in $\Delta ;$ and so on. Hence in general:

$$
E=\Sigma e_{i} A_{i}+\Sigma e_{i} e_{i} A_{i j}+\Sigma e_{i} e_{i} e_{k} A_{i ; k}+\ldots,
$$

the suffixes in each summation running from 1 to $n^{2}$. (This result follows at once from Taylor's Theorem, $A_{i}, A_{i}, A_{i j k}, \ldots$ being partial derivatives of $\Delta$.) It is to be observed that none of the errors occur squared or to higher powers in (1).

§3. Range of error. If $\left|e_{i}\right| \leqslant \epsilon_{i}$, we deduce from (1):

$$
|E| \leqslant \Sigma \epsilon_{i}\left|A_{i}\right|+\Sigma \epsilon_{i} \epsilon_{i}\left|A_{i j}\right|+\Sigma \epsilon_{i} \epsilon_{i} \epsilon_{k}\left|A_{i j k}\right|+\ldots .
$$

vertical bars denoting absolute values. If we put $\epsilon_{i}=\epsilon$, and neglect $\epsilon^{2},(2)$ becomes

$$
|E| \leqslant \epsilon \Sigma\left|A_{i}\right| \text {. }
$$

Thus $\Sigma\left|A_{i}\right|$ may be regarded as a measure of the sensitivity of a determinant to small errors whose squares may be neglected.

As an application of these formulae, consider the first determinant in $\S 1$, and suppose that the range of error in each element is $\pm \frac{1}{2}$. We find $\Sigma\left|A_{i}\right|=33099, \Sigma\left|A_{i j}\right|=2 \Sigma \mid a_{i}=970$, and (for any third order determinant) $\Sigma\left|A_{i j k}\right|=6$. Hence (3) gives $\pm 16 \tilde{5} 50$ as a first approximation to the range of error in $\Delta$. More accurately, (4) gives:

$$
|E| \leqslant \frac{1}{2} .33099+\frac{1}{4} \cdot 970+\frac{1}{*} .6<16793 .
$$

If we suppose that the determinant has occurred in some practical calculation, and that the elements as given are only rough first approximations (with $\epsilon=\frac{1}{2}$ ), then the important question arises: To what further degree of accuracy $\eta$ should the elements be evaluated, in order that the error in $\Delta$ may not exceed a given limit, say for example $0 \cdot 2$ ?

We have in general, when the range of error in each element is $\pm \eta$,

$$
|E| \leqslant \eta \Sigma\left|B_{i}\right|+\eta^{2} \Sigma\left|B_{i j}\right|+\ldots \text {, }
$$

where $B_{i}, B_{i}, \ldots$ are the correct values of the minors to which $A_{i}, A_{i}, \ldots$ are approximations. 
Now

and hence

$$
\begin{gathered}
\left|A_{i}-B_{i}\right|=\text { error in minor of } a_{i} \\
\leqslant \epsilon \sum_{j}\left|A_{i j}\right|+\epsilon^{2} \sum_{j k}\left|A_{i j k}\right|+\ldots,
\end{gathered}
$$

$$
\left|B_{i}\right| \leqslant\left|A_{i}\right|+\epsilon \sum_{j}\left|A_{i j}\right|+\epsilon^{2} \sum_{j k}\left|A_{i j k}\right|+\ldots
$$

Summing for $i=1, \ldots, n$,

Similarly,

$$
\Sigma\left|B_{i}\right| \leqslant \Sigma\left|A_{i}\right|+\epsilon \Sigma\left|A_{i j}\right|+\epsilon^{2} \Sigma\left|A_{i j k}\right|+\ldots
$$

Hence

$$
\Sigma\left|B_{i j}\right| \leqslant \Sigma\left|A_{i j}\right|+\epsilon \Sigma\left|A_{i j k}\right|+\ldots
$$

$$
\begin{aligned}
|E| \leqslant & \eta\left[\Sigma\left|A_{i}\right|+\epsilon \Sigma\left|A_{i j}\right|+\epsilon^{2} \Sigma\left|A_{i j k}\right|+\ldots\right] \\
& +\eta^{2}\left[\Sigma\left|A_{i j}\right|+\epsilon \Sigma\left|A_{i j k}\right|+\ldots .\right]+\ldots
\end{aligned}
$$

This formula gives the range of error in a determinant when the elements are correct to within $\pm \eta$, in terms of the minors in a first approximation in which the elements are correct to within $\pm \epsilon$.

Let us apply this to the determinant already considered, for which

$$
\Sigma\left|A_{i}\right|+\epsilon \Sigma\left|A_{i j}\right|+\epsilon^{2} \Sigma\left|A_{i j k}\right|=\mathbf{3 3 5 8 5 \cdot 5} .
$$

Putting $\eta=\cdot 000005$, we find $|E|<0 \cdot 17$. Thus, in order that the error in $\Delta$ should not exceed 0.2 , it is sufficient to evaluate each element to 5 decimal places.

§4. Probability Distribution. Denote the probability distribution of $e_{i}$ by $p_{i}\left(e_{i}\right)$, and the $r^{\text {th }}$ moment of this function by

$$
m_{r i} \equiv \int_{-\infty}^{\infty} e_{i}^{r} p_{i}\left(e_{i}\right) d e_{i} .
$$

The functions $p_{i}$ being given, we can assume that these moments $m_{r i}$ are known; in terms of them we can calculate the $r^{\text {th }}$ moment $M_{r}$ of $P(E)$. We have in fact:

$$
M_{r} \equiv \int_{-\infty}^{\infty} E^{r} P(E) d E=\int_{-\infty}^{\infty} \ldots \int_{-\infty}^{\infty} E^{r} . \prod_{1}^{n^{2}}\left[p_{i}\left(e_{i}\right) d e_{i}\right] .
$$

On the right hand side of (5), $E^{r}$ is to be interpreted as a function of $e_{i}$, by means of (1). Consequently, for any value of $r$, we can expand $E^{r}$ and integrate term by term, making use of (4), and of the con$\operatorname{ditions} \int_{-\infty}^{\infty} p_{i} d e_{i}=1$. 
For example,

$$
E^{2}=\Sigma A_{i}^{2} e_{i}^{2}+2 \Sigma A_{i} A_{j} e_{i} e_{j}+2 \Sigma A_{i} A_{i j} e_{i}^{2} e_{j}+2 \Sigma A_{i} A_{j k} e_{i} e_{j} e_{k}+\ldots
$$

On integration this gives

$\Sigma A_{i}^{2} m_{2 i}+2 \Sigma A_{i} A_{j} m_{1 i} m_{1 j}+2 \Sigma A_{i} A_{i j} m_{2 i} m_{1 j}+2 \Sigma A_{i} A_{j k} m_{1 i} m_{1 j} m_{1 k}+\ldots$ as the value of $M_{2}$.

The given functions have been assumed to be symmetrical; i.e. $p_{i}\left(e_{i}\right)=p_{i}\left(-e_{i}\right) ;$ consequently the odd moments $m_{1 i}, m_{3 i}, \ldots$ all vanish. We need, then, only take account of those terms of $E^{r}$ which contain only even powers of the errors $e_{i}$; all other terms vanish on integration. We then find

$M_{1}=0$

$M_{2}=\Sigma A_{i}^{2} m_{2 i}+\Sigma A_{i j}^{2} m_{2 i} m_{2 j}+\Sigma A_{i j k}^{2} m_{2 i} m_{2 j} m_{2 k}+\ldots \ldots$,

$M_{3}=6 \Sigma A_{i} A_{j} A_{i j} m_{2 i} m_{2 j}+6 \Sigma A_{i j} A_{j k} A_{i k} m_{2 i} m_{2 j} m_{2 k}+6 \Sigma A_{i} A_{j k} A_{i j k} m_{2 i} m_{2 j} m_{2 k}+\ldots$,

$M_{4}=\Sigma A_{i}^{4} m_{4 i}+6 \Sigma A_{i}^{2} A_{j}^{2} m_{2 i} m_{2 j}+\ldots \ldots$

and so on.

Each of these expressions has only a finite number of terms, but they rapidly become very complicated. However, if the original errors are known to be sufficiently small, i.e. if $p_{i}$ are practically zero except where $e_{i}$ are small, then the moments $m_{r i}$ are also small quantities; we can therefore approximate to $M_{r}$ successfully with only a few terms, and the succeeding moments $M_{r}$ will rapidly diminish in order of magnitude.

With a change of notation, equation (7) can be rewritten:

$$
S^{2}=\Sigma A_{i}^{2} \sigma_{i}^{2}+\Sigma A_{i j}^{2} \sigma_{i}^{2} \sigma_{j}^{2}+\Sigma A_{i j k}^{2} \sigma_{i}^{2} \sigma_{j}^{2} \sigma_{k}^{2}+\ldots
$$

giving the standard deviation $S\left(=\sqrt{ } M_{2}\right)$ of the determinant in terms of the standard deviations $\sigma_{i}\left(=\sqrt{ } m_{\Omega i}\right)$ of the elements.

Having thus found $M_{1}, M_{2}, M_{3}, \ldots$, we can approximate to $P(E)$ in one of the usual ways. For instance, if we assume that the errors $e_{i}$ follow the normal law of distribution,

$$
p_{i}\left(e_{i}\right)=\frac{1}{\sigma_{i} \sqrt{2 \pi}} \exp \left(-e_{i}^{2} / 2 \sigma_{i}^{2}\right)
$$

(or, more generally, that they follow a symmetrical law of Charlier's Type $A$ ), then the law for the determinant must necessarily be of Charlier's Type $A$. We can thus assume

$$
P(E)=\left[1+A \frac{d^{3}}{d E^{3}}+B \frac{d^{4}}{d E^{4}}+\ldots\right] \frac{1}{S \sqrt{2 \pi}} e^{-E^{*} / 2 S^{*}} .
$$


$S$ is given by (8), and the other constants by:

$$
A=-\frac{M_{3}}{3 !}, \quad B=\frac{M_{4}-3 S^{4}}{4 !}, \text { etc. }
$$

these results being found by comparing the moments of the function with $M_{2}, M_{3}, \ldots$.

To a first approximation,

$$
S^{2}=\Sigma A_{i}^{2} \sigma_{i}^{2} ; A, B, \ldots=0 .
$$

To a second approximation,

$$
\begin{aligned}
S^{2} & =\Sigma A_{i}^{2} \sigma_{i}^{2}+\sum A_{i}^{2} A_{j}^{2} \sigma_{i}^{2} \sigma_{j}^{2} ; \\
A & =-\Sigma A_{i} A_{j} A_{i j} \sigma_{i}^{2} \sigma_{j}^{2} ; \\
4 ! B & =\sum A_{i}^{4} m_{4 i}+6 \Sigma A_{i}^{2} A_{j}^{2} \sigma_{i}^{2} \sigma_{j}^{2}-3\left(\Sigma A_{i}^{4} \sigma_{i}^{4}+2 \Sigma A_{i}^{2} A_{j}^{2} \sigma_{i}^{2} \sigma_{j}^{2}\right) \\
& =0,
\end{aligned}
$$

since for normal distributions $m_{4 i}=3 \sigma_{i}^{4}$.

For practical purposes a more important case arises when the elements have been calculated correct to $x$ places of decimals, the last figure being forced, i.e. the last digit retained is increased by 1 when the first digit not retained is $5,6,7,8$ or 9 . We may take it that all errors between $\pm \frac{1}{2} 10^{-\infty}$ are equally likely, and that none exceed these limits. Remembering that $\int_{-\infty}^{\infty} p(e) d e$ must be unity, we have

$$
\begin{aligned}
p(e) & =10^{x}, & & \text { if }-\frac{1}{2} 10^{-x} \leqslant e<\frac{1}{2} 10^{-x}, \\
& =0, & & \text { otherwise; }
\end{aligned}
$$

the same law of distribution applies to all the elements.

Then we have

$$
\begin{aligned}
m_{1} & =m_{3}=\ldots=0 \\
m_{2} & =\int_{-\frac{1}{2} 10^{-x}}^{\frac{1}{2} 10^{-x}} e^{2} \cdot 10^{x} d e=\frac{1}{12 \cdot 10^{2 x}} \\
m_{4} & =\int_{-\frac{1}{2} 10^{-x}}^{\frac{1}{2} 10^{-x}} e^{4} \cdot 10^{x} d e=\frac{1}{80 \cdot 10^{4 x}} \\
M_{1} & =0 \\
M_{2} & =\frac{\Sigma A_{i}^{2}}{12 \cdot 10^{2 x}}+\frac{\Sigma A_{i j}^{2}}{144 \cdot 10^{4 x}}+\ldots ; \\
M_{3} & =\frac{\Sigma A_{i} A_{i} A_{i j}}{24 \cdot 10^{4 x}}+\ldots \\
M_{4} & =\frac{\Sigma A_{i}^{4}}{80 \cdot 10^{4 x}}+\frac{\Sigma A_{i}^{2} A_{j}^{2}}{24.10^{4 x}}+\ldots
\end{aligned}
$$


Hence, neglecting terms involving $10^{-6 x}$, we find:

$P(E)=\left[1-\frac{\Sigma A_{i} A_{j} A_{i j}}{144.10^{4 x}} \frac{d^{3}}{d E^{3}}-\frac{\Sigma A_{i}^{4}}{288.10^{5 x}} \frac{d^{4}}{d E^{4}}\right] \frac{1}{S \sqrt{2 \pi}} e^{-E^{2} / 2 S^{2}}$,

where

$$
S^{2}=\frac{\sum A_{i}^{2}}{12 \cdot 10^{2 x}}+\frac{\sum A_{i j}^{2}}{144 \cdot 10^{4 x}} .
$$

As an application of these formulae, consider the determinant:

$$
\left|\begin{array}{ccc}
\sqrt{ } 7 & \sqrt{ } 2 & \sqrt{ } 2 \\
\sqrt{ } 2 & \sqrt{ } 11 & \sqrt{ } 3 \\
\sqrt{ } 3 & \sqrt{ } 5 & \sqrt{ } 6
\end{array}\right|,
$$

the value of which, to five places, is 6.93899 . Evaluating each element to two places, we get:

$\begin{array}{lll}2.65 & 1.41 & 1.41 \\ 1.41 & 3.32 & 1.73 \\ 1.73 & 2.24 & 2.45\end{array}$

the value of which, to five places, is 6.98983 . For the latter determinant we find (correct to the number of places given in each case):

$$
\begin{array}{ll}
\Sigma\left|A_{i}\right| & =26 \cdot 8, \\
\Sigma A_{i}^{2} & =111 \cdot 9, \\
\Sigma A_{i j}^{2} & =78, \\
\Sigma A_{i} A_{j} A_{i j} & =287, \\
\Sigma A_{i}^{4} & =1078, \\
\epsilon & =\cdot 005 .
\end{array}
$$

By (3), the maximum range of error is $\pm \cdot 135$. By (10), the standard deviation (to a first approximation) is 031 . These results may be compared with the actual error $\cdot 051$. The second approximation to the probability distribution is given by:

$$
S=\cdot 0314, \quad A=-\cdot 0002, \quad B=-\cdot 0004 .
$$

§5. Error in quotient of determinants. Consider the quotient $\frac{\Delta_{1}}{\Delta_{2}}$ of the determinants ${ }^{1}$ :

$$
\begin{aligned}
& \Delta_{1}=\left|\begin{array}{lllll}
a_{i} & a_{i 1} & a_{i 2} & \ldots & a_{i n-1}
\end{array}\right|, \quad(i=1 \ldots n)
\end{aligned}
$$

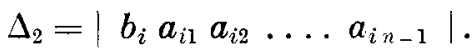

1 The notation means that the determinants have these as $i^{\text {th }}$ rows. The determinants are identical except in their first columns. As pointed out in $\$ 1$, such quotients occur in the solution of a set of $n$ simultaneous linear equations. 
Suppose that the elements $a_{i}, b_{i}, a_{i j}$ are subject to errors $e_{i}, f_{i}, e_{i j}$; and that the resultant errors in $\Delta_{1}, \Delta_{2}, \frac{\Delta_{1}}{\Delta_{2}}$ are $E_{1}, E_{2}, E$. Form the determinant

$$
\begin{aligned}
& \Delta_{3}=\left|c_{i} a_{i 1} a_{i 2} \ldots a_{i n-1}\right|, \\
\text { where } & c_{1}=a_{i}-X b_{i}, \\
X & =\text { the calculated value of } \frac{\Delta_{1}}{\Delta_{2}} ; \\
\text { so that } & \Delta_{3}=\text { the calculated value of } \Delta_{1}-X \Delta_{2}=0 .
\end{aligned}
$$

The correct value of $\Delta_{1}-X \Delta_{2}$ is, however, not zero.

Let $\quad g_{i}=$ error in $c_{i}=e_{i}-X f_{i}$,

$$
\begin{aligned}
E_{3} & =\text { error in } \Delta_{3}=E_{1}-X E_{2}, \\
A_{i} & =\text { minor of } a_{i} \text { in } \Delta_{1} \\
& =\text { minor of } b_{i} \text { in } \Delta_{2} \\
& =\text { minor of } c_{i} \text { in } \Delta_{3}, \\
A_{i j} & =\text { minor of } a_{i j} \text { in } \Delta_{1}, \\
B_{i j} & =\text { minor of } a_{i j} \text { in } \Delta_{2}, \\
C_{i j} & =\text { minor of } a_{i j} \text { in } \Delta_{3}=A_{i j}-X B_{i j} .
\end{aligned}
$$

It follows from (11) that the minors of any one row of $\Delta_{3}$ are proportional to the minors of the first row, i.e.

$$
C_{i j}=\frac{A_{i} C_{1 j}}{A_{1}} .
$$

Using (1), we have as first approximations,

$$
\begin{aligned}
& E_{2}=\Sigma f_{i} A_{i}+\Sigma e_{i j} B_{i j}, \\
& E_{3}=\Sigma g_{i} A_{i}+\Sigma e_{i j} C_{i j} .
\end{aligned}
$$

Correct to the second order,

$$
\begin{aligned}
E & =\frac{\Delta_{1}+E_{1}}{\Delta_{2}+E_{2}}-\frac{\Delta_{1}}{\Delta_{2}} \\
& =\frac{\Delta_{2} E_{1}-\Delta_{1} E_{2}}{\Delta_{2}\left(\Delta_{2}+E_{2}\right)} \\
& =\frac{E_{1}-X E_{2}}{\Delta_{2}}\left(1+\frac{E_{2}}{\Delta_{2}}\right)^{-1} \\
& =\frac{E_{3}}{\Delta_{2}}\left(1-\frac{E_{2}}{\Delta_{2}}\right) .
\end{aligned}
$$


Thus, to the first order,

$$
E=\frac{1}{\Delta_{2}}\left[\Sigma\left(e_{i}-X f_{i}\right) A_{i}+\Sigma e_{i j} C_{i, j}\right]
$$

To the second order,

$$
\begin{aligned}
E & =\frac{E_{3}}{\Delta_{2}}-\frac{1}{\Delta_{2}^{2}}\left[\Sigma f_{i} A_{i}+\Sigma e_{i j} B_{i j}\right]\left[\Sigma\left(e_{i}-X f_{i}\right) A_{i}+\Sigma e_{i j} C_{i j}\right] \\
E^{2} & =\frac{1}{\Delta_{2}^{2}}\left[\Sigma\left(e_{i}-X f_{i}\right) A_{i}+\Sigma e_{i j} C_{i j}\right]^{2}
\end{aligned}
$$

§6. Range of error in quotient of determinants. Suppose

$$
\left|e_{i}\right| \leqslant \epsilon_{i}, \quad\left|f_{i}\right| \leqslant \zeta_{i}, \quad\left|e_{i j}\right| \leqslant \epsilon_{i,}
$$

From (13), the range of $E$ is given to a first approximation by:

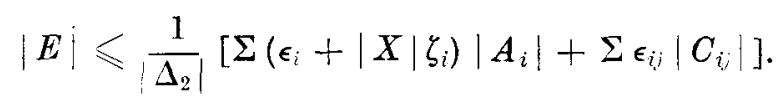

If the given ranges are all equal, i.e., $\epsilon_{i}=\zeta_{i}=\epsilon_{i,}=\epsilon$, we have, using (12)

$$
\begin{aligned}
E \mid & \leqslant \frac{\epsilon}{\left|\Delta_{2}\right|}\left[(1+|X|) \Sigma\left|A_{i}\right|+\Sigma\left|\frac{A_{i} C_{1 ;}}{A_{1}}\right|\right] \\
& \leqslant \frac{\epsilon}{\left|\Delta_{2} A_{1}\right|} \Sigma A_{i} \cdot\left[(1+|X|)\left|A_{1}\right|+\Sigma\left|C_{1 j}\right|\right] .
\end{aligned}
$$

This can be expressed in the form

$$
|E| \leqslant \epsilon \frac{1+\mid X}{\left|\Delta_{2} A_{1}\right|} \mid S_{1} S_{2},
$$

where $S_{1}=$ sum of absolute values of first minors of first column of $D$,

$S_{2}=$ sum of absolute values of first minors of first row of $D$, $D$ being the determinant $\left|\frac{a_{i}-X b_{i}}{1+|X|} \quad a_{i 1} a_{i 2} \ldots a_{i n-1}\right|$.

§7. Probability distribution for quotient of determinants. Let the standard deviations of $e_{i}, f_{i}, e_{i}$, be $\sigma_{i}, \tau_{i}, \sigma_{i}$, it being assumed that the first moments are zero. Let $M_{1}, M_{2}, M_{3} \ldots$ be the moments of the probability distribution $P(E)$ of $E$. Using (14) and (15), proceeding as in $\$ 4$, and remembering that in accordance with (6) the 
first moment of $E_{3}$ is zero, we get, correct to the second order in the deviations :

$$
\begin{aligned}
& M_{1}=\frac{1}{\Delta_{2}^{2}}\left[X \Sigma \tau_{i}^{2} A_{i}^{2}-\Sigma \sigma_{i j}^{2} B_{i j} C_{i j}\right], \\
& M_{2}=\frac{1}{\Delta_{2}^{2}}\left[\Sigma\left(\sigma_{i}^{2}+X^{2} \tau_{i}^{2}\right) A_{i}^{2}+\Sigma \sigma_{i j}^{2} C_{i j}^{2}\right], \\
& M_{3}=\ldots=0 .
\end{aligned}
$$

Thus, assuming that the required function is of Charlier's Type $A$, we have as a first approximation:

$$
P(E)=\frac{1}{S \sqrt{2 \pi}} e^{-(E-a)^{2} / 2 S^{2}},
$$

where

$$
\begin{aligned}
a & =M_{1}=\left[X \Sigma \tau_{i}^{2} A_{i}^{2}-\Sigma \sigma_{i j}^{2} B_{i j} C_{i j}\right] / \Delta_{2}^{2}, \\
S^{2} & =M_{2}-M_{1}^{2}=\left[\Sigma\left(\sigma_{i}^{2}+X^{2} \tau_{i}^{2}\right) A_{i}^{2}+\Sigma \sigma_{i j}^{2} C_{i j}^{2}\right] / \Delta_{2}^{2} .
\end{aligned}
$$

The second approximation to $P(E)$ involves many complicated summations.

If the given standard deviations are all equal, i.e. $\sigma_{i}=\tau_{i}=\sigma_{i j}=\sigma$, we have, using (12):

$$
\begin{aligned}
a & =\frac{\sigma^{2}}{\Delta_{2}^{2}}\left[X \Sigma A_{i}^{2}-\frac{1}{A_{1}} \Sigma B_{i j} A_{i} C_{1 j}\right], \\
S^{2} & =\frac{\sigma^{2}}{\Delta_{2}^{2} A_{1}^{2}} \Sigma A_{i}^{2} \cdot\left[\left(1+X^{2}\right) A_{1}^{2}+\Sigma C_{1 j}^{2}\right] .
\end{aligned}
$$

As in $\S 6$, we note that (18) can be written

$$
S^{2}=\sigma^{2} \frac{1+X^{2}}{\Delta_{2}^{2} A_{1}^{2}} S_{3} S_{4},
$$

where $S_{3}=$ sum of squares of first minors of first column of $D^{\prime}$,

$S_{4}=$ sum of squares of first minors of first row of $D^{\prime}$, $D^{\prime}$ being the determinant $\left|\frac{a_{i}-X b_{i}}{\left(1+X^{2}\right)^{\frac{1}{2}}} a_{i 1} a_{i 2} \ldots a_{i n-1}\right|$.

As an application of these formulae, consider the value of $X$ found from the equations

$$
\begin{aligned}
& X \sqrt{ } 7+Y \sqrt{ } 2+Z \sqrt{ } 2=\sqrt{ } 17 \\
& X \sqrt{ } 3+Y \sqrt{ } 11+Z \sqrt{ } 3=-\sqrt{ } 2 \\
& X \sqrt{ } 2+Y \sqrt{ } 5+Z \sqrt{ } 6=-\sqrt{ } 3
\end{aligned}
$$


To four places, the value is $X=3 \cdot 1468$. Let us however approximate by evaluating each coefficient to two places. We then have:

$$
\begin{aligned}
& \Delta_{1}=\left|\begin{array}{rrr}
4 \cdot 12 & 1 \cdot 41 & 1 \cdot 41 \\
-1 \cdot 41 & 3 \cdot 32 & 1 \cdot 73 \\
-1 \cdot 73 & 2 \cdot 24 & 2 \cdot 45
\end{array}\right|=21 \cdot 842244, \\
& \Delta_{2}=\left|\begin{array}{rrr}
2 \cdot 65 & 1 \cdot 41 & 1.41 \\
1.41 & 3 \cdot 32 & 1.73 \\
1 \cdot 73 & 2 \cdot 24 & 2 \cdot 45
\end{array}\right|=6.989832 .
\end{aligned}
$$

The following results are correct to the number of places given in each case:

$$
\begin{aligned}
& X=\frac{\Delta_{1}}{\Delta_{2}}=3 \cdot 1249 \\
& \Delta_{3}=\left|\begin{array}{lll}
-4 \cdot 16 & 1 \cdot 41 & 1 \cdot 41 \\
-5 \cdot 82 & 3 \cdot 32 & 1 \cdot 73 \\
-7 \cdot 14 & 2 \cdot 24 & 2 \cdot 45
\end{array}\right|=\cdot 01 \\
& A_{1}=4 \cdot 26 \\
& \Sigma\left|A_{i}\right|=6 \cdot 8 \\
& \Sigma A_{i}^{2}=23 \cdot 25 \\
& \Sigma B_{i j} A_{i} C_{1 j}=-264, \\
& (1+|X|)\left|A_{1}\right|+\Sigma\left|C_{1 j}\right|=30 \\
& X \Sigma A_{i}^{2}-\frac{1}{A_{1}} \Sigma B_{i j} A_{i} C_{1 j}=134, \\
& \left(1+X^{2}\right) A_{1}^{2}+\Sigma C_{1 j}^{2}=313, \\
& \epsilon=\cdot 005 \\
& \sigma^{2}=\frac{1}{12.10^{4}} .
\end{aligned}
$$

From (16), (17) and (18) we deduce that the range of error is \pm 035 , and that $a=\cdot 00002, \quad S=\cdot 008$. The actual error is $\cdot 022$.

§8. Error in an arbitrary function. The method of $\$ 4$ may also be used to determine the probability distribution of error in an arbitrary function $F$ (instead of the determinant $\Delta$ ) of the quantities $a_{i}$. Equation (1) is then formally the same, but, since $F_{i i}=\partial^{2} F / \delta a_{i}^{2} \neq 0$, it includes terms with higher powers of the errors $e_{i}$. The following are the corresponding results, suffixes of $F$ indicating partial derivatives. 
If the given laws of distribution are symmetrical,

$$
\begin{aligned}
& M_{1}=\frac{1}{2} \Sigma F_{i i} m_{2 i}+\frac{1}{24} \Sigma F_{i i i} m_{4 i}+\frac{1}{4} \sum F_{i i j} m_{2 i} m_{2 j}+\ldots \\
& M_{2}=\Sigma F_{i}^{2} m_{2 i}+\frac{1}{4} \sum F_{i i}^{2} m_{4 i}+\Sigma\left(F_{i i} F_{i j}+F_{i j}^{2}\right) m_{2 i} m_{2 j}+\ldots \ldots ; \\
& M_{3}=\frac{3}{2} \Sigma F_{i}^{2} F_{i i} m_{4 i}+\left(\frac{3}{2} \Sigma F_{i}^{2} F_{j i}+6 \sum F_{i} F_{j} F_{i j}\right) m_{2 i} m_{2 j}+\ldots \ldots ; \\
& M_{4}=\Sigma F_{i}^{4} m_{4 i}+6 \Sigma F_{i}^{2} F_{j}^{2} m_{2 i} m_{2 j}+\ldots \ldots \quad(i \neq j) .
\end{aligned}
$$

If the given laws of distribution are normal, the required function will be of the form

$$
P(E)=\left[1+A \frac{d^{3}}{d E^{3}}+B \frac{d^{4}}{d E^{4}}+\ldots\right] \frac{1}{S \sqrt{2 \pi}} e^{-(B-a)^{2} / 2 S^{2}} .
$$

The constants are given by

$$
a=M_{1}, S^{2}=M_{2}-M_{1}^{2}, A=-\frac{M_{3}}{6}+\frac{M_{1} M_{2}}{2}-\frac{M_{1}^{3}}{3}, B=\frac{M_{4}}{24}+\frac{M_{1}^{4}}{12}-\frac{M_{2}^{2}}{8}+M_{1} A .
$$

To a first approximation

$$
a=\frac{1}{2} \Sigma F_{i i} \sigma_{i}^{2} ; \quad S^{2}=\Sigma F_{i}^{2} \sigma_{i}^{2} ; \quad A, B, \ldots=0 .
$$

To a second approximation

$$
\begin{aligned}
& a=\frac{1}{2} \sum F_{i i} \sigma_{i}^{2}+\frac{1}{8} \sum F_{i i i i} \sigma_{i}^{4}+\frac{1}{4} \sum F_{i i j j} \sigma_{i}^{2} \sigma_{j}^{2} \\
& S^{2}=\sum F_{i}^{2} \sigma_{i}^{2}+\frac{1}{2} \sum F_{i i}^{2} \sigma_{i}^{4}+\Sigma F_{i j}^{2} \sigma_{i}^{2} \sigma_{j}^{2} \\
& A=-\frac{1}{2} \sum F_{i i} F_{i}^{2} \sigma_{i}^{4}-\Sigma F_{i j} F_{i} F_{j} \sigma_{i}^{2} \sigma_{j}^{2} \\
& B, \ldots=0
\end{aligned}
$$

If the arguments of $F$ have been calculated correct to $x$ decimal places, the last figure being forced, then the second approximation is given by:

$$
\begin{aligned}
& a=\frac{\Sigma F_{i i}}{24 \cdot 10^{2, i}}+\frac{\Sigma F_{i i i i}}{1920 \cdot 10^{4 x}}+\frac{\Sigma F_{i i j i}}{576 \cdot 10^{4 x}} ; \\
& S^{2}=\frac{\Sigma F_{i}^{2}}{12 \cdot 10^{2 x}}+\frac{\Sigma F_{i i}^{2}}{720 \cdot 10^{4 x}}+\frac{\Sigma F_{i i}}{288 \cdot 10^{4 x}}+\frac{\Sigma F_{i j}^{2}}{144 \cdot 10^{4 x}} ; \\
& A=-\frac{\sum F_{i i} F_{i}^{2}}{720 \cdot 10^{4, x}}-\frac{\sum F_{i j} F_{i} F_{j}}{144 \cdot 10^{4 x}} \\
& B=-\frac{\sum F_{i}^{4}}{2880 \cdot 10^{4}} \text {. }
\end{aligned}
$$

